\title{
EDUCAÇÃO EMPREENDEDORA: UM ESTUdO DE CASO NO CURSO DE ENGENHARIA AEROESPACIAL
}

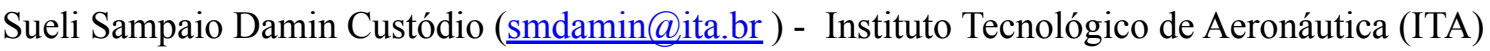

Christopher Shneider Cerqueira (hris@ita.br) - Instituto Tecnológico de Aeronáutica (ITA)

\section{Resumo}

Este artigo expõe os resultados do processo educacional denominado Projeto Integrador, que contemplou a integração parcial entre as disciplinas obrigatórias de Direito, de Engenharia de Sistemas e de Projeto e Construção de Sistemas Aeroespaciais no curso de Engenharia Aeroespacial (AESP). Apoiado nos quatro pilares da UNESCO que fundamentam a aprendizagem do século XXI (saber conhecer, saber fazer, saber ser e saber conviver), o estudo de caso da aplicação das metodologias Educação Empreendedora (EE) e Aprendizagem Baseada em Projetos (PBL) se direciona aos interessados em explorar a transdisciplinaridade e o ensino da inovação e empreendedorismo inseridos no curso de Graduação. A pesquisa envolveu estudantes regularmente matriculados nos cursos de graduação, e os resultados evidenciam a EE e o PBL como ferramentas válidas para o aprimoramento da formação dos estudantes de engenharia, sobretudo no desenvolvimento de competências e habilidades exigidas nas Diretrizes Curriculares Nacionais para os cursos de Engenharia (DCNs).

Palavras chave: transdisciplinaridade; educação empreendedora; ensino da inovação; aprendizado baseado em projetos; engenharia aeroespacial. 


\section{INTRODUÇÃO}

Com a transformação digital das organizações, emergem questionamentos sobre as mudanças, o uso e as aplicações de tecnologias e as relações que se desenvolvem e impulsionam a inserção de novas competências e habilidades do profissional de engenharia. As mudanças se estendem para além do mundo do trabalho e trazem impactos não só no modo de produção e manufatura, mas também na maneira com a qual a formação do engenheiro se estabelece. Há exigências de um novo profissional, flexível, atento às mudanças de seu tempo, ou seja, conectado aos desafios do séc. XXI e aos ecossistemas de inovação conforme expõe Bittencourt (2019).

Apoiado nos quatro pilares da UNESCO (DELORS, 2012) que fundamentam a aprendizagem do século XXI - saber conhecer, saber fazer, saber ser e saber conviver - o presente trabalho se direciona tanto para aqueles que já lidam com o processo educativo, quanto para os que estão interessados em explorar a transdisciplinaridade, o ensino da inovação e o empreendedorismo educacional (FAYOLLE, 2007) inseridos no curso de Gradução de Engenharia, mais especificamente na Engenharia Aeroespacial conforme Sillitto et al. (2018). Nessa medida, o presente artigo expõe a sistemática de trabalho denominada Projeto Integrador, que contemplou a integração parcial entre as disciplinas obrigatórias de Direito, Engenharia de Sistemas e de Projeto e Construção de Sistemas Aeroespaciais no curso de Graduação em Engenharia Aeroespacial (AESP).

O Projeto Integrador introduziu, nos primeiros semestres de 2020 e 2021, os métodos conhecidos como Educação Empreendedora (EE) e Project-Based Learning (PBL) para as turmas dos primeiros semestres do $3^{\circ}$ ano do curso da AESP. Envolveu 3 professores, 2 mentoras, 8 palestrantes e 40 alunos. Esse Projeto Acadêmico contemplou três objetivos gerais: (i) desenvolver atividades práticas que promovessem o engajamento dos estudantes para o trabalho em equipes, e, sobretudo, para a resolução de problemas e projetos complexos; (ii) ampliar as habilidades e competências profissionais desejáveis à prática da engenharia; e (iii) identificar formas de se melhorar a educação superior em empreendedorismo e inovação (BITTENCOURT, 2019; LIÑÁN, 2007).

Considerando a aplicação das metodologias EE (FRAGOSO; ROCHA-JUNIOR; XAVIER, 2019) e PBL (CASALE, 2013; CASALE et al., 2011; FAUST; PAULSON, 1998) no curso de Graduação, é esperado que os resultados deste trabalho possam contribuir para a 
melhoria da educação do ensino superior e ao mesmo tempo evidenciar a EE e o PBL como ferramentas úteis para a formação dos estudantes de engenharia. Para fins de orientação ao leitor, o trabalho está seccionado em: (1) Introdução; (2) Referenciais Teóricos adotados no Projeto Integrador implementado; (3) Metodologia proposta; (4) Análise e discussão dos resultados parciais obtidos e (5) Conclusões acompanhadas das limitações do estudo e das reflexões para trabalhos futuros.

\section{FUNDAMENTOS TEÓRICOS}

Segundo Boahin e Hofman (2014), as empresas e as organizações públicas cada vez mais buscam engenheiros que sejam capazes de combinar habilidades e competências de forma inovadora para lidar com os desafios de um ambiente globalizado. É com base neste contexto, que a experiência do projeto integrador se insere, ou seja, do reconhecimento do papel das instituições de ensino superior (IES) na criação de um ambiente acadêmico que seja capaz de contribuir para o ecossistema de inovação.

Os desafios apresentados estimularam os estudantes a apreender conceitos, conhecimentos e técnicas que os ajudassem a resolver problemas do dia a dia da missão proposta, com os quais eles terão de lidar na vida profissional no setor aeroespacial, ou seja, descrever as necessidades, mapear os stakeholders e entender o contexto da missão. Buscou-se com isso, formar profissionais que soubessem planejar, buscar informações e interações, estabelecer metas e indicadores, e que fossem criativos, autônomos e tivessem uma visão sistêmica da missão.

A partir da compreensão dos estudantes como atores no processo de formação, o projeto integrador introduziu métodos educacionais para estimular os alunos a trabalhar em equipes multidisciplinares, a ter capacidade de aprender, identificar, modelar e resolver problemas, ter conhecimento da legislação pertinente à missão e adaptar-se a situações novas e complexas como, por exemplo, dimensionar e integrar recursos físicos, humanos e financeiros visando melhorias contínuas, projetar ou aperfeiçoar sistemas e processos levando em consideração as comunidades envolvidas.

A base teórica da Educação Empreendedora, mais especificamente os 4 Pilares da Educação baseados no Relatório da UNESCO da Comissão Internacional sobre Educação para o Século XXI, coordenada por Delors (2012), ocupou posição estratégica no 
planejamento, definição de objetivos e ações no processo educacional implementado conforme tabela abaixo:

TABELA 1 - Informações sobre o projeto integrador.

\begin{tabular}{|l|l|l|}
\hline $\begin{array}{l}\text { P Pilares da } \\
\text { Educação- Unesco }\end{array}$ & Objetivos & Ações \\
\hline Aprender a fazer & $\begin{array}{l}\text { Implementar projetos } \\
\text { integradores no curso de Eng. } \\
\text { Aeroespacial }\end{array}$ & $\begin{array}{l}\text { Integração parcial das disciplinas } \\
\text { de Direito, Engenharia de } \\
\text { Sistemas e Projeto de Sistemas } \\
\text { Espaciais com o intuito de } \\
\text { introduzir habilidades que } \\
\text { pudessem colocar o } \\
\text { conhecimento em prática }\end{array}$ \\
\hline $\begin{array}{l}\text { Aprender a viver com } \\
\text { os outros }\end{array}$ & $\begin{array}{l}\text { Criar um ativo de networking } \\
\text { com os Ex-alunos e instituições } \\
\text { no setor aeroespacial }\end{array}$ & $\begin{array}{l}\text { Debates abertos à comunidade } \\
\text { para tratar de temas voltados à } \\
\text { carreira e às trajetórias } \\
\text { profissionais, destacando a } \\
\text { importância da empatia e } \\
\text { liderança }\end{array}$ \\
\hline Aprender a conhecer & $\begin{array}{l}\text { Criar um ciclo de eventos com a } \\
\text { apresentação da área de Inovação } \\
\text { e Empreendedorismo }\end{array}$ & $\begin{array}{l}\text { Apresentação de desafios e } \\
\text { problemas para incentivar a } \\
\text { tomada de decisão, o estímulo à } \\
\text { pesquisa e compreensão de } \\
\text { cenários }\end{array}$ \\
\hline Aprender a ser & $\begin{array}{l}\text { Criar um ciclo de debates sobre } \\
\text { Direito e Política Espacial }\end{array}$ & $\begin{array}{l}\text { Debates abertos para incentivar a } \\
\text { autonomia, o pensamento crítico, } \\
\text { a cidadania e a responsabilidade } \\
\text { ética do engenheiro }\end{array}$ \\
\hline
\end{tabular}

Fonte: Os autores

Os métodos introduzidos com base na EE proporcionaram aos estudantes a compreensão de cenários contextualizados para enfrentar situações que fazem parte do cotidiano e da futura carreira profissional (BOUD; FELETTI, 1999). Esses métodos inserem a perspectiva de uma visão humanística e holística conforme prescrevem os artigos $3^{\circ}$ e $4^{\circ}$ das Diretrizes Curriculares da Engenharias (DCNs), além de integrarem a noção de empresa-escola, e de valorizarem a transdisciplinaridade por meio da ênfase a atividades orientadas por projetos.

\section{METODOLOGIA}

Nos primeiros semestres de 2020 e 2021, a integração de disciplinas se deu por meio de projetos associados ao setor aeroespacial com base em Ackoff (1994) e Wertz, Everett e Puschell (2011). Em 2020, foram quatro desafios relacionados a serviços espaciais e direcionados a temas como meteorologia, coleta de dados, observação da terra e 
telecomunicações. Em 2021, os projetos estiveram associados ao Programa Artemis, desenvolvido pela Nasa, que conta com parceiros comerciais e internacionais para a exploração do sistema solar. As missões lunares levarão a primeira mulher à superfície lunar e contemplam objetivos estratégicos com a criação da Plataforma Orbital Lunar Gateway que servirá de centro de comunicações movido a energia solar e ao mesmo tempo servirá de módulo de habitação e laboratório de ciências para a exploração do Sistema Solar. Cada disciplina definiu objetivos e aprendizados específicos. Esta seção expõe um breve descritivo do projeto integrador, seus objetivos, metodologias, e, principalmente, os desafios sobre a adaptação para o formato virtual nos anos de 2020 e 2021, dando destaque aos desafios de 2021.

TABELA 2 - Informações quantitativas sobre o alcance do projeto.

\begin{tabular}{|c|c|c|}
\hline Ano/Semestre & $\begin{array}{c}\text { Número de alunos } \\
\text { envolvidos }\end{array}$ & Número de Projetos \\
\hline 2021.1 & 21 & 04 \\
\hline 2020.1 & 19 & 04 \\
\hline & 40 & 08 \\
\hline
\end{tabular}

Fonte: Os autores

O propósito geral da integração consistiu em desenvolver ações voltadas à inserção de metodologias ativas na Graduação (ESCRIVÃO FILHO; RIBEIRO, 2009). Para tanto, o projeto se estruturou da seguinte forma: (1) foram organizados 4 grupos de cerca de 5 pessoas; (2) foram apresentados desafios direcionados ao Programa Artemis e (3) os grupos estavam matriculados nas mesmas disciplinas com integração parcial de conteúdo.

As disciplinas técnicas introduziram os desafios envolvendo Engenharia de Sistemas e Projeto e Construção de Sistemas Espaciais. A primeira apresentou o processo no qual o engenheiro aeroespacial vai, a partir da coleta de necessidades, organizar e direcionar o desenvolvimento de alternativas de soluções que atendam as métricas apontadas pela coleta das necessidades e, nesse ínterim, derivar arquiteturas funcionais e físicas, descrever os conceitos de operação, estruturar o fluxo temporal de atividades que o sistema deve realizar, de forma que possam ser levantados o máximo de informações nas primeiras fases de concepção, antecipando demandas futuras. A segunda apresentou o domínio de um problema com o qual o engenheiro aeroespacial deve lidar, as motivações para o desenvolvimento de um sistema espacial, as características dos serviços derivados de sistemas espaciais, a definição de uma missão espacial e sua decomposição ao nível dos componentes e equipamentos utilizados para a construção do sistema. 
Nas edições que foram realizadas as integrações, os alunos usaram o conteúdo de uma disciplina para complementar a outra (FORTESCUE; STARK; SWINERD, 2011; INTERNATIONAL COUNCIL ON SYSTEMS ENGINNERING - INCOSE, 2015). Da disciplina de Projeto e Construção de Sistemas Espaciais, os termos e elementos específicos de uma missão espacial eram usados como vocabulário para as alternativas de soluções que eram dadas em Engenharia de Sistemas (SIS), e da disciplina de SIS, o processo de iterativamente desdobrando os níveis da complexidade do sistema. Os temas dos projetos dos alunos foram previamente acordados para a realização do alinhamento dos tópicos semanais e do direcionamento dos exercícios de exploração da temática espacial por meio do processo da Engenharia de Sistemas.

Em 2021, foram tratados quatro projetos baseados no padrão CubeSat, com as missões a serem realizadas ao redor da Lua e relacionadas com o Programa Artemis, sendo eles: (i) demonstração tecnológica de capacidade de locomoção para o ponto lagrangiano a partir da Lua; (ii) utilização de constelação de coleta de dados de Internet das Coisas para roteamento de informação e apoio aos astronautas; (iii) coleta de dados do clima espacial lunar e (iv) experimentação de crescimento de microrganismos na lua. Nesta edição, os alunos receberam missões mais específicas.

No primeiro projeto, o CubeSat deveria deslocar-se de um ponto da órbita da lua para o ponto lagrangiano, e para isso o grupo precisou explorar as tecnologias de propulsão e as funcionalidades necessárias para o controle da trajetória até a posição desejada.

No segundo projeto, um grupo de CubeSats deveria coletar informações de equipamentos do tipo internet das coisas que poderiam estar distribuídos no solo lunar, dando apoio aos astronautas para retransmissão de mensagens de emergência, e na própria interação com as "coisas" distribuídas pelo solo lunar. Para isso, o grupo precisou explorar arranjos de constelações ao redor da Lua, mecanismos de manutenção de órbita devido aos diferentes gradientes de gravidade lunar, e estratégias de roteamento de informação entre satélites.

No terceiro projeto, o CubeSat deveria coletar dados do clima espacial lunar de forma a iniciar a criação de um mapa de condições espaciais nas regiões de baixa órbita lunar. Para realizar essa missão, o grupo também precisou estudar mecanismos de manutenção de órbita, e os sensores utilizados para cumprir as funções de coleta das informações desejadas pelos cientistas. 
No quarto projeto, o CubeSat deveria manter as condições de um experimento de crescimento de microrganismos que seriam colocados em solo lunar, de forma a monitorar a possível proliferação dos microrganismos. Para isso, o grupo precisou explorar estratégias de pouso em solo lunar, alternativas para manter o condicionamento ambiental para realização do experimento e formas de mensurar os indicadores da evolução do experimento. Cada um dos grupos partiu de uma missão espacial geral com quatro segmentos: (i) lançador, responsável por levar o artefato até seu local de operação; (ii) espacial, responsável por realizar a operação no espaço; (iii) solo, responsável por se comunicar com os elementos do segmento espacial e (iv) usuário, responsável por interagir com os clientes da missão.

Em todas as missões foram realizadas três macro-atividades do ciclo de vida: (i) entendimento do Problema; (ii) concepção de Alternativas Conceituais e (iii) proposta de Arquitetura Concretizada. No Entendimento do Problema, foi abordado o tipo espacial e a arquitetura de referência conhecida, por meio do entendimento do que era demandado pelos interessados e impactados pela missão. Após a identificação dos stakeholders, identificou-se também os requisitos legais para o cumprimento da missão e estabeleceu-se a integração com a disciplina de Direito (BITTENCOURT NETO, 2011).

$\mathrm{Na}$ Concepção de Alternativas conceituais, foram levantadas as principais funções do sistema e a dinâmica do comportamento dos possíveis arranjos conceituais. Na Proposta de Arquitetura Concretizada, foram apresentados os desdobramentos tecnológicos, e seus comportamentos, para que fossem escolhidas opções para realizar a arquitetura conceitual conservada por meio das análises da engenharia de sistemas, bem como por meio do entendimento da Arquitetura Jurídico-Institucional. O propósito foi gerenciar a viabilidade da missão, bem como identificar as restrições legais da missão, o risco de impactos e danos a partir da compreensão da cadeia de responsabilidade e atores envolvidos (PEREIRA, 2000).

\section{RESULTADOS E DISCUSSÃO}

O Estudo GUESSS/Brasil (Global University Entrepreneurial Spirit Students' Survey), aplicado em 2013/2014, apresentou estudos comparativos de instituições de ensino superior (IES) no mundo todo. Este estudo apontou os interesses e motivações dos estudantes pelas carreiras escolhidas, bem como o aumento de universidades que têm integrado o programa de empreendedorismo às suas propostas curriculares e criado um ambiente acadêmico propício 
ao surgimento de startups e empreendimentos por parte dos estudantes. O estudo contemplou 12.561 estudantes e mais de 60 instituições brasileiras de ensino superior e, ao final, apresentou orientações e recomendações. Destaca-se a orientação para a integração de diferentes disciplinas dos cursos a partir de uma perspectiva transversal de EE, por meio da inserção da área da inovação, a identificação e exploração de oportunidades e a avaliação de riscos.

Há também estudos que usam a Teoria do Comportamento Planejado (TCP), desenvolvida por Ajzen (1987, 1991), para analisar o impacto da EE sobre a escolha de carreira dos estudantes, e se o tipo de curso impacta a intenção empreendedora e potenciais empreendedores no grupo de estudantes (FAYOLLE, 2007). Esses estudos têm ganhado relevância nas últimas décadas e têm mapeado problemas nos processos educativos, sobretudo em relação às competências, habilidades e ao perfil do egresso desejado.

Buscando identificar o impacto da EE sobre a intenção empreendedora dos estudantes (AJZEN, 1991), bem como o interesse por atividades e disciplinas na área de inovação e empreendedorismo, foi encaminhado questionário para os estudantes com a colaboração do Centro Acadêmico (CASD). A IES em que foi realizado o estudo de caso tem 647 estudantes matriculados nos cursos de Graduação de Engenharia, sendo que 88 responderam, ou seja, $13,6 \%$ do universo desses estudantes. Deste percentual, 18,2\%, no momento do estudo, tem previsão de formatura em 2021 (T.21), 6,7\% tem previsão de formatura em 2022 (T22), 25\% em 2023 (T.23), 19,3\% em 2024 (T.24) e 31,8\% em 2025 (T.25). A amostra analisada remete aos 88 estudantes regularmente matriculados nos cursos de graduação que receberam formulários online visando mensurar qual ou quais fatores foram considerados relevantes no momento da escolha pela carreira em engenharia, além de mensurar o interesse pelas áreas de inovação e empreendedorismo.

Considerando o distanciamento social devido à pandemia de COVID-19, optou-se por encaminhar o link do questionário online aos estudantes. A amostra não foi obtida randomicamente, pois o questionário foi respondido pelos estudantes que se interessaram pelo convite. Entretanto, a amostra aponta aspectos relevantes para a proposta de melhoria da EE nos cursos de Engenharia, sobretudo para atender o interesse dos estudantes por atividades e conteúdos voltados à criação de Programa de Educação Empreendedora (Educational Entrepreneurship Program- EEP). 
TABELA 3 - Distribuição por curso.

\begin{tabular}{|c|c|}
\hline Cursos & Número de respondentes \\
\hline Aeroespacial & 14 \\
\hline Aeronáutica & 17 \\
\hline Civil & 8 \\
\hline Computação & 21 \\
\hline Eletrônica & 14 \\
\hline Mecânica & 14 \\
\hline
\end{tabular}

Fonte: Dados da Pesquisa/CASD (2021)

Segundo as respostas obtidas, há demanda por educação em empreendedorismo. A pesquisa mostrou que $11 \%$ se interessam pela área do empreendedorismo, $10 \%$ têm interesse em criar uma startup, e $6 \%$ em atuar em Fintechs (startups que trabalham para inovar e otimizar serviços no sistema financeiro). Em outras palavras, $27 \%$ se interessam pela área (Figuras 1 e 2). Os resultados mostram também a intenção dos estudantes em empreender. Dos 88 que responderam, 9,1\% informam que já estão empreendendo, 28,4\% indicam que planejam empreender no futuro próximo e 39,8\% expõem que, embora não tenham interesse em empreender, têm interesse em conhecer e participar de atividades associadas à educação empreendedora. Apenas 14\% indicam interesse em atuar na área de Engenharia, excluindo a área de Computação.

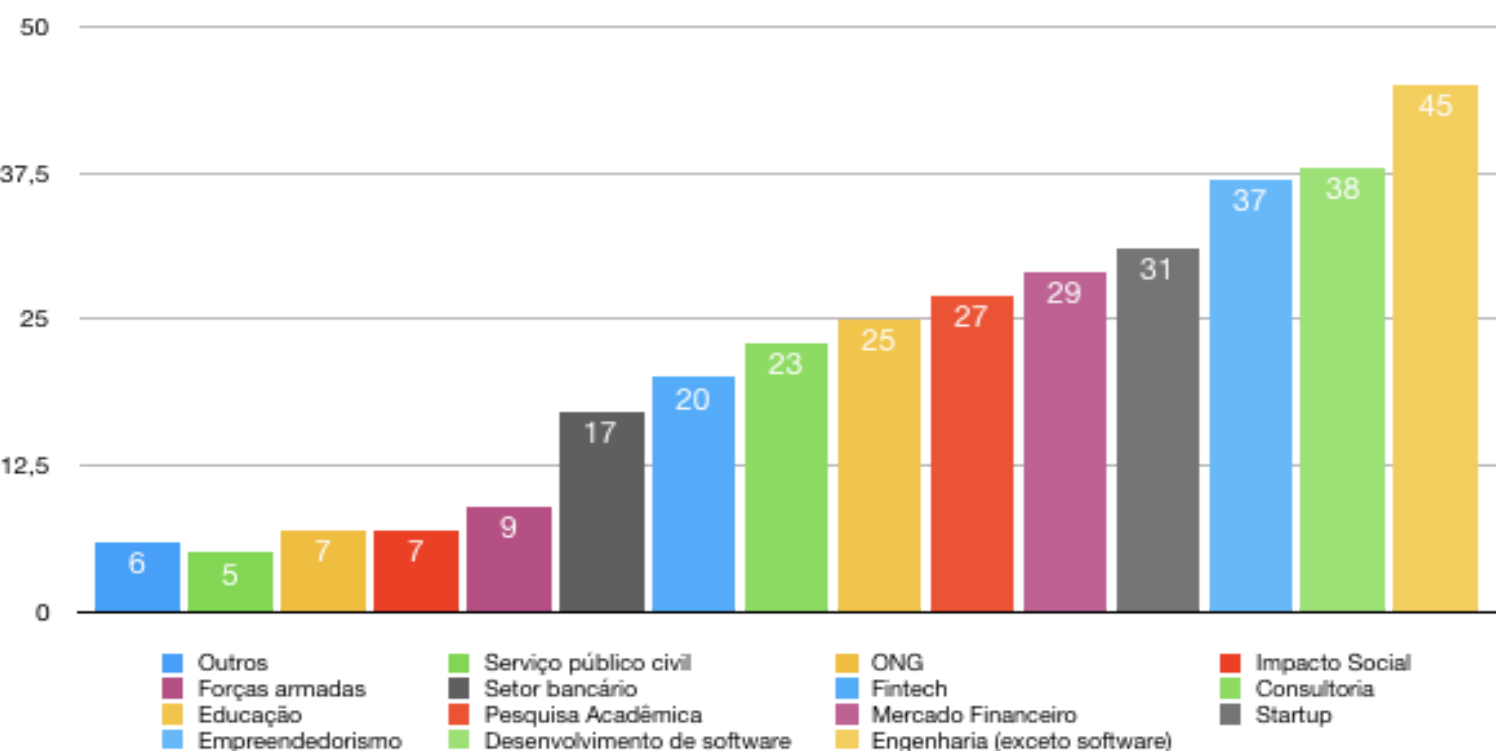

FIGURA 1 - Expectativa de atuação dos Respondentes. Fonte: Dados da Pesquisa/CASD (2021)

Quando se analisou as respostas dos estudantes da AESP, observou-se pouca variação, mas um maior interesse em empreender. A pesquisa mostrou que 14\% se interessam pela área 
do empreendedorismo, 11\% têm interesse em criar uma startup, e 9\% em atuar em Fintech, ou seja, 34\% se interessam pela área (Figuras 2).

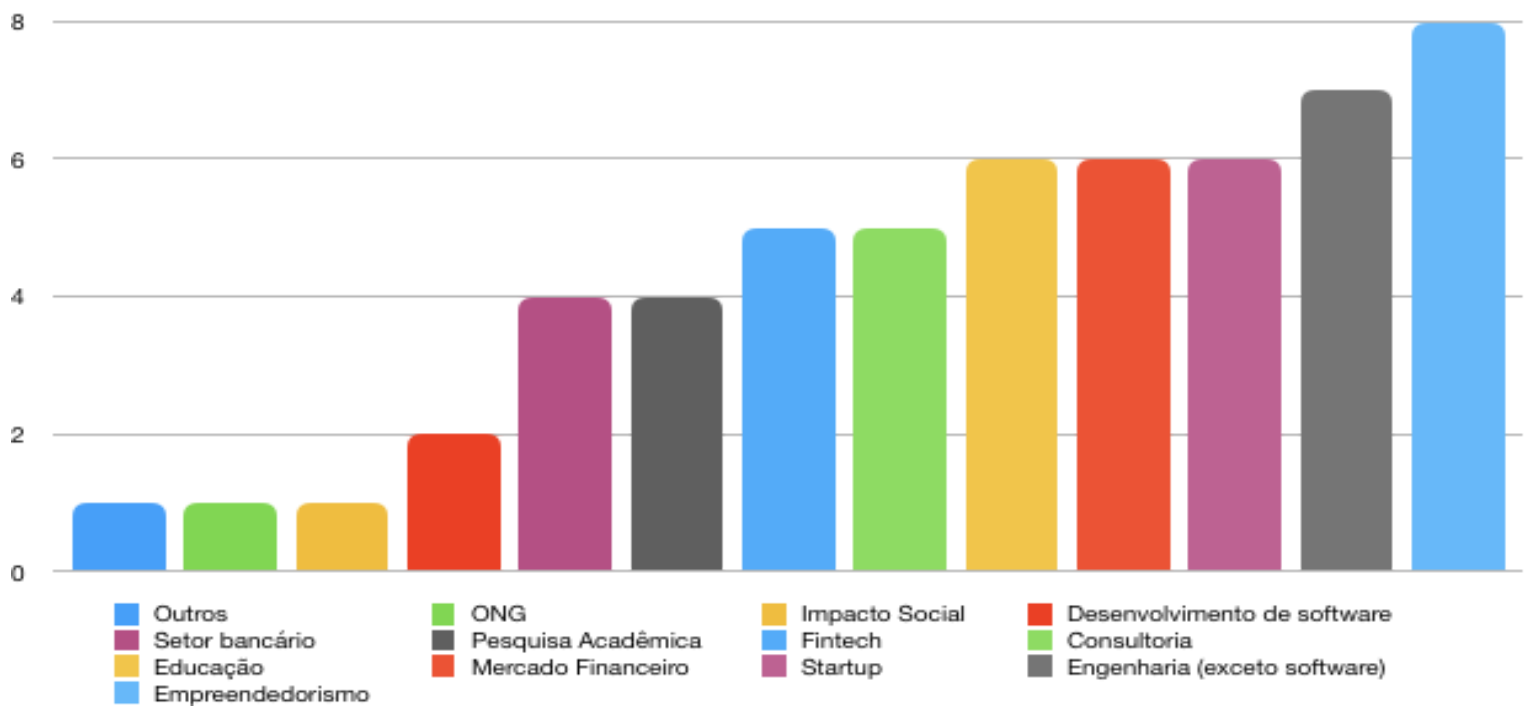

FIGURA 2 - Expectativa de atuação dos alunos Eng. AESP. Fonte: Dados da Pesquisa/CASD (2021)

O resultado parcial do projeto sugere que a proposta implementada impulsionou não apenas a compreensão de problemas técnicos, mas também as restrições legais da missão. Os feedbacks aos professores envolvidos foram positivos tanto em 2020 quanto em 2021, com destaque para a capacidade de tomar decisões tendo um entendimento maior sobre os riscos. Nessa medida, a proposta parece ter propiciado aos estudantes uma experiência próxima da realidade de áreas mais complexas e de gerenciamento de riscos da Engenharia.

Contudo, as dinâmicas presenciais foram impactadas por conta do cenário da COVI-19. A instituição optou por adotar o Google Workspace for Education, com acesso a um conjunto de ferramentas e serviços adaptados ao ensino remoto. Desde então, foram produzidos e carregados eventos, videoaulas e atividades em grupo na plataforma Google for education com o intuito de garantir aos estudantes acesso amplo durante todo o período de realização do projeto, além de plataformas, softwares e métodos específicos direcionados ao setor aeroespacial, como Data flow concept operations (CONOPS).

\section{CONCLUSÃO}

Este artigo tratou da aplicação de metodologias ativas e educação empreendedora no curso de Engenharia Espacial e seus desafios, dentre eles, o que seria possível integrar, levando-se em conta as respectivas ementas, o intervalo temporal de um semestre para 
executar o projeto e o cenário da COVID. As limitações identificadas na aplicação foram de três ordens: (i) planejamento de atividades com a necessidade dos docentes se apropriarem minimamente dos componentes curriculares das disciplinas envolvidas e sobretudo, das metodologias de aprendizagem utilizadas em cada disciplina; (ii) design dos desafios com o dimensionamento da complexidade diante do prazo estabelecido e (iii) a adaptação das dinâmicas e do ensino presencial para o ensino remoto. A experiência da aplicação trouxe um aprendizado significativo para os professores e impulsionou uma nova modelagem do design dos desafios para um cenário de médio e longo prazos (pós-pandêmico), envolvendo stakeholders do ecossistema de inovação no setor aeroespacial, com a parceria estratégica da Nasa.

Os estudantes envolvidos foram ouvidos e as percepções registradas na pesquisa evidenciaram o alto grau de interesse pelas áreas de inovação e empreendedorismo, especialmente pelo desenvolvimento de atividades hands-on por meio de dinâmicas de grupos, com a finalidade de promover o engajamento, o conhecimento e a descoberta. Os resultados obtidos apontaram para o efeito positivo da EE sobre a intenção empreendedora dos estudantes. Nessa medida, o aprendizado por PBL, viabilizado pela integração parcial das disciplinas de Direito, Engenharia de Sistemas e Projeto e Construção de Sistemas Aeroespaciais, pode ser um dos caminhos a ser adotado pela IES para inserir a cultura da inovação em suas propostas curriculares e cumprir as exigências das DCNs para os cursos de Engenharia.

Ressalta-se, contudo, que os resultados não podem ser generalizados, uma vez que foram obtidos por aplicação restrita a duas turmas de engenharia, além de ter ocorrido em período que foge à normalidade, ou seja, os anos de 2020 e 2021 afetados pela pandemia de COVID. Com o propósito de ampliar a base de dados colhidos, os professores optaram por continuar com o projeto em 2022, envolvendo novos desafios associados ao Programa Artemis.

\section{REFERÊNCIAS}

ACKOFF, R.L. Systems thinking and thinking systems. System Dynamics Review., v.10, p. 175-188, 1994. AJZEN, I. Attitudes, traits, and actions: dispositional prediction of behavior in social psychology. In: BERKOWITZ, L. (Ed.), Advances in experimental social psychology. San Diego: Academic Press, 1987. v. 20, p. 1-6.

AJZEN, I. The Theory of planned behavior. Organizational Behavior and Human Decision Processes, v. 50 , n.2, p. 179-211, 1991.

BITTENCOURT, A. C.. A influência das práticas empreendedoras de uma universidade na formação de 
ecossistemas de inovação: um estudo à luz da teoria do trabalho institucional. 2019. Tese (Doutorado), Universidade do Vale dos Sinos, São Leopoldo, Brasil, 2019.

BITTENCOURT NETO, O. Direito espacial contemporâneo: responsabilidade internacional. Curitiba: Juruá, 2011.

BOAHIN, P.; HOFMAN,W. Perceived effects of competency-based training on the acquisition of professional skills. International Journal of Educational Development, v.36, p. 81-89, 2014.

BOUD, D.; FELETTI, G. Changing-problem learning. In: BOUD, D.; FELETTI, G. (Ed.). The Challenge of problem-based learning. London, Kogan Page, 1999, p. 1-14.

CASALE, A. et al. Mapas cognitivos na avaliação da aprendizagem baseada em problemas. Revista Portuguesa de Educação, v. 24, n. 2, p. 243-263, 2011.

CASALE, A. Aprendizagem baseada em problemas - desenvolvimento de competências para o ensino de engenharia. Tese (Doutorado) - Departamento de Engenharia de Produção da Escola de Engenharia de São Carlos, Universidade de São Paulo. São Paulo. 2013.

DELORS, J. (Org.). Educação um tesouro a descobrir - relatório para a Unesco da comissão internacional sobre educação para o Século XXI. 7.ed.. São Paulo: Cortez, 2012.

ESCRIVÃO FILHO, E.; RIBEIRO, L. R. C. Aprendendo com PBL - aprendizagem baseada em problema: relato de uma experiência em cursos de Engenharia da EESC-USP. Revista Minerva, v. 6, p. 23-30, 2009.

FAUST, J.L, PAULSON, D.R. Active learning in the college classroom. Journal on Excellence in College Teaching, v. 9, n 2, p. 3-24, 1998.

FAYOLLE, A. (Ed.). Handbook of research in entrepreneurship education - a general perspective. Cheltenham: Edward Elgar, 2007. v.1.

FORTESCUE, P. \& STARK; J. \& SWINERD, G.G. Spacecraft systems engineering, 4th ed. New York: Wiley, 2011.

FRAGOSO, R.; ROCHA-JUNIOR, W.; XAVIER, A. Determinant factors of entrepreneurial intention among university students in Brazil and Portugal. Journal of Small Business \& Entrepreneurship, v.32, n.2, 2169-2610, 2019.

INTERNATIONAL COUNCIL ON SYSTEMS ENGINEERING. Systems engineering handbook: a guide for system life cycle processes and activities. New York: Wiley, 2015.

KRUEGER, N.; CARSRUD, A. Entrepreneurial intentions: applying the theory of planned behavior. Entrepreneurship \& Regional Development, v. 5, n. 4, p.315-330, 1993.

LIÑÁN, F.. The Role of entrepreneurship education in the entrepreneurial process. In: A. Fayolle (Ed.), Handbook of research in entrepreneurship education - a general perspective. Cheltenham, Northampton: Edward Elgar, 2007. Vol. 1, p. 230-247,

PEREIRA, L. C. R. Ensaio sobre a responsabilidade internacional do estado e suas consequências no direito internacional: a saga da responsabilidade internacional do Estado. São Paulo: Ltr, 2000.

SILLITTO, H. et al. Envisioning systems engineering as a transdisciplinary venture. In: INCOSE INTERNATIONAL SYMPOSIUM, 28, 2018. Washington. DOI: 10.1002/j.2334-5837.2018.00529.x.

WERTZ, J. R.; EVERETT, D. F.; \& PUSChELL, J. J.. Space mission engineering: the new SMAD. Hawthorne: Microcosm Press, 2011. 